\title{
Communication
}

\section{Effect of Vitamins Other than Vitamin C on Viruses: Virus-Inactivating Activity of Vitamin $\mathbf{K}_{5}$}

\author{
Akira Murata, Shigeru SaKaI, Kumio OdA, \\ Kazumasa NAKATSUMI, and \\ Fumio KATO ${ }^{1}$ \\ Laboratory of Applied Microbiology, \\ Department of Agricultural Chemistry, \\ Saga University, \\ Saga 840, Japan
}

(Received June 24, 1983)

Key Words virus, phage, vitamin $\mathrm{K}_{5}$, 4-amino-2-methyl-1-naphthol

We have to date systematically studied the inactivation in vitro of viruses by vitamin $\mathrm{C}$ (ascorbic acid), using a wide variety of bacterial viruses (phages) as a model system (1-5). The study has led to the conclusion that the virus-inactivating activity in vitro of vitamin $\mathrm{C}$ is a generally observable phenomenon in all kinds of viruses (6). In contrast, very little has been studied of the effect on viruses of vitamins other than vitamin $\mathrm{C}$.

We have made a systematic study of the effect on viruses of a number of watersoluble vitamins and their related substances, and found that vitamin $\mathrm{K}_{5}$ (4-amino2-methyl-1-naphthol) has an inactivating activity on a wide variety of phages.

Phages used are listed in Table 1 . These 21 phages present a wide variety from the viewpoint of nucleic acid type, morphology, host bacteria, serological grouping and certain other characteristics. Vitamins and their related substances examined are listed in Table 2.

The 20 vitamins and related substances other than vitamin $\mathrm{K}_{5}$ exerted no significant inactivating effect on any of the phages tested.

In contrast, vitamin $\mathrm{K}_{5}$ exerted an inactivating effect on all of the phages tested, although the phages exhibited differential sensitivities to the inactivation by vitamin $\mathrm{K}_{5}$. Table 3 shows the inactivation rates of the 21 phages in certain concentrations of vitamin $\mathrm{K}_{5}$. At $1 \times 10^{-3} \mathrm{M}$, full inactivation of 11 phages $(\mathrm{J} 1, \mathrm{~T} 1$, $\lambda, \mathrm{M} 2, \mathrm{~T} 3, \phi \mathrm{X} 174, \mathrm{MS} 2, \mathrm{GA}, \mathrm{Q} \beta, \mathrm{SP}$ and $\phi 6)$ was achieved and of 6 phages (T1, $\lambda$, MS2, GA, Q $\beta$ and $\phi 6)$ at $3 \times 10^{-4} \mathrm{M}$. Sensitivities of the phages to vitamin $\mathrm{K}_{5}$ were arranged, from the highest to the lowest, in the following order: GA, $\phi 6, \mathrm{Q} \beta, \mathrm{MS} 2$,

1 村田 晃, 酒井 繁, 小田久美男, 中津海和正, 加藤富民雄 
Table 1. List of bacterial viruses (phages) used.

\begin{tabular}{|c|c|c|c|}
\hline Phage & Nucleic acid & Morphology & Host \\
\hline $\begin{array}{l}\mathrm{J} 1 \\
\phi 219 \\
\mathrm{~T} 1, \mathrm{~T} 5, \lambda\end{array}$ & \multirow{3}{*}{$\begin{array}{c}\text { Double-stranded } \\
\text { DNA }\end{array}$} & & $\begin{array}{l}\text { Lactobacillus casei } \\
\text { Lactobacillus plantarum } \\
\text { Escherichia coli }\end{array}$ \\
\hline $\begin{array}{l}\text { J2, NHc } \\
\phi 16 \\
\text { T2, T4 } \\
\text { SP10, SP01, M2 }\end{array}$ & & & $\begin{array}{l}\text { Lactobacillus casei } \\
\text { Lactobacillus plantarum } \\
\text { Escherichia coli } \\
\text { Bacillus subtilis }\end{array}$ \\
\hline $\mathrm{T} 3$ & & & Escherichia coli \\
\hline$\phi \times 174$ & \multirow{2}{*}{$\begin{array}{c}\text { Single-stranded } \\
\text { DNA }\end{array}$} & 8 & Escherichia coli \\
\hline$\delta \mathrm{A}$ & & & Escherichia coli \\
\hline $\mathrm{MS} 2, \mathrm{GA}, \mathrm{Q} \beta, \mathrm{SP}$ & $\begin{array}{l}\text { Single-stranded } \\
\text { RNA }\end{array}$ & $\square$ & Escherichia coli \\
\hline$\phi 6$ & $\begin{array}{c}\text { Double-stranded } \\
\text { RNA }\end{array}$ & مי & Pseudomonas phaseolicola \\
\hline
\end{tabular}

Table 2. Vitamins and their related substances examined.

\begin{tabular}{lclc}
\hline \multicolumn{1}{c}{ Trivial name } & Vitamin name & \multicolumn{1}{c}{ Trivial name } & Vitamin name \\
\hline Thiamine & $\mathrm{B}_{1}$ & p-Aminobenzoic acid & $\mathrm{B}_{\mathbf{X}}$ \\
Riboflavin & $\mathrm{B}_{2}$ & Choline & \\
Nicotinamide & $\mathrm{B}_{3}$ & $\begin{array}{l}\text { Lipoic acid } \\
\text { Inositol }\end{array}$ & \\
Nicotinic acide & & $d$-Biotin & $\mathrm{H}$ \\
$d$-Pantothenic acid & $\mathrm{B}_{5}$ & 4-Amino-2-methyl-1- & $\mathrm{K}_{5}$ \\
Pyridoxine & $\mathrm{B}_{6}$ & naphthol & \\
Cyanocobalamin & $\mathrm{B}_{12}$ & Anthranilic acid & $\mathrm{L}_{1}$ \\
Orotic acid & $\mathrm{B}_{13}$ & Hesperidin & $\mathrm{P}$ \\
Pangamic acid & $\mathrm{B}_{15}$ & Rutin & $\mathrm{U}$ \\
Folic acid & $\mathrm{B}_{\mathrm{C}}$ & Cabagin & \\
Carnitine & $\mathrm{B}_{\mathrm{T}}$ & &
\end{tabular}

SP, T1, $\lambda, \mathrm{J} 1, \phi \mathrm{X} 174, \mathrm{M} 2, \mathrm{~T} 3, \delta \mathrm{A}, \phi 219, \mathrm{SP} 10, \mathrm{SP} 01, \phi 16, \mathrm{~J} 2, \mathrm{NHc}, \mathrm{T} 5, \mathrm{~T} 2, \mathrm{~T} 4$.

These results indicate that vitamin $\mathrm{K}_{5}$ has a potential virus-inactivating activity. In other words, the virus-inactivating activity could be a new biological activity of vitamin $\mathrm{K}_{5}$. 
Table 3. Inactivating activity of vitamin $\mathrm{K}_{5}$ on a wide variety of phages.

Phages $\left(1 \sim 4 \times 10^{7} \mathrm{PFU} / \mathrm{ml}\right)$ were incubated with vitamin $\mathrm{K}_{5}$ in $0.02 \mathrm{M}$ Tris- $\mathrm{HCl}$ buffer (pH 7.4) for $30 \mathrm{~min}$ at $37^{\circ} \mathrm{C}$. Survival of the control without the vitamin was represented as $100 \%$.

\begin{tabular}{|c|c|c|c|}
\hline \multirow{3}{*}{ Phage } & \multicolumn{3}{|c|}{ Survival $(\%)$} \\
\hline & \multicolumn{3}{|c|}{ Concn. (M) of $\mathrm{K}_{5}$} \\
\hline & $1 \times 10^{-3}$ & $3 \times 10^{-4}$ & $1 \times 10^{-4}$ \\
\hline $\mathbf{J} 1$ & 0 & 1 & 20 \\
\hline$\phi 219$ & 4 & 15 & 65 \\
\hline $\mathrm{T} 1$ & 0 & 0 & 15 \\
\hline T5 & 30 & 45 & 75 \\
\hline$\lambda$ & 0 & 0 & 20 \\
\hline $\mathrm{J} 2$ & 20 & 30 & 55 \\
\hline $\mathrm{NHc}$ & 25 & 40 & 80 \\
\hline$\phi 16$ & 15 & 25 & 55 \\
\hline $\mathrm{T} 2$ & 45 & 60 & 80 \\
\hline $\mathrm{T} 4$ & 60 & 75 & 100 \\
\hline SP10 & 5 & 15 & 65 \\
\hline SP01 & 5 & 15 & 70 \\
\hline M2 & 0 & 4 & 40 \\
\hline T3 & 0 & 5 & 45 \\
\hline$\phi \times 174$ & 0 & 3 & 40 \\
\hline$\delta \mathrm{A}$ & 1 . & 4 & 55 \\
\hline MS2 & 0 & 0 & 7 \\
\hline $\mathrm{GA}$ & 0 & 0 & 2 \\
\hline $\mathrm{Q} \beta$ & 0 & 0 & 4 \\
\hline SP & 0 & 0 & 10 \\
\hline$\phi 6^{\mathrm{a}}$ & 0 & 0 & 3 \\
\hline
\end{tabular}

${ }^{a}$ Phage $\phi 6$ was incubated for $20 \mathrm{~min}$ at $25^{\circ} \mathrm{C}$ because of its relative instability at $37^{\circ} \mathrm{C}$.

This work was presented at the Annual Meeting of the Vitamin Society of Japan, Kyoto, June 3, 1977.

\section{REFERENCES}

1) Murata, A., Kitagawa, K., and Saruno, R. (1971): Inactivation of bacteriophages by ascorbic acid. Agric. Biol. Chem., 35, 294-296.

2) Murata, A., Kitagawa, K., Inmaru, H., and Saruno, R. (1972): Inactivation of singlestranded DNA and RNA phages by ascorbic acids and thiol reducing agents. Agric. Biol. Chem., 36, 2597-2599.

3) Murata, A., and Kitagawa, K. (1973): Mechanism of inactivation of bacteriophage J1 by ascorbic acid. Agric. Biol. Chem., 37, 1145-1151.

4) Murata, A., Oyadomari, R., Ohashi, T., and Kitagawa, K. (1975): Mechanism of Vol. 29, No. 6, 1983 
inactivation of bacteriophage $\delta$ A containing single-stranded DNA by ascorbic acid. $J$. Nutr. Sci. Vitaminol., 21, 261-269.

5) Murata, A., and Uike, M. (1976): Mechanism of inactivation of bacteriophage MS2 containing single-stranded RNA by ascorbic acid. J. Nutr. Sci. Vitaminol., 22, 347-354.

6) Murata, A. (1975): Virucidal activity of vitamin C: Vitamin C for prevention and treatment of viral diseases, in Proc. 1st. Intersect. Congr. Internat. Assoc. Microbiol. Soc., Vol. 3, ed. by Hasegawa, T., Science Council of Japan, Tokyo, pp. 432-436. 